\title{
Detection of acute cerebral hemorrhage in rabbits by magnetic induction
}

\author{
J. Sun ${ }^{1}$, G. Jin ${ }^{1}$, M.X. Qin ${ }^{1}$, Z.B. Wan ${ }^{2}$, J.B. Wang ${ }^{3}$, C. Wang ${ }^{3}$, W.Y. Guo ${ }^{3}$, L. Xu ${ }^{1}$, X. Ning ${ }^{1}$, J. Xu ${ }^{1}$, \\ X.J. Pu ${ }^{1}$, M.S. Chen ${ }^{1}$ and H.M. Zhao ${ }^{2}$ \\ ${ }^{1}$ College of Biomedical Engineering and Medical Imaging, Third Military Medical University, Chongqing, China \\ ${ }^{2}$ Experimental Animal Center, Third Military Medical University, Chongqing, China \\ ${ }^{3}$ College of Electronic Engineering, Xidian University, X'an, China
}

\begin{abstract}
Acute cerebral hemorrhage $(\mathrm{ACH})$ is an important clinical problem that is often monitored and studied with expensive devices such as computed tomography, magnetic resonance imaging, and positron emission tomography. These devices are not readily available in economically underdeveloped regions of the world, emergency departments, and emergency zones. We have developed a less expensive tool for non-contact monitoring of $\mathrm{ACH}$. The system measures the magnetic induction phase shift (MIPS) between the electromagnetic signals on two coils. ACH was induced in 6 experimental rabbits and edema was induced in 4 control rabbits by stereotactic methods, and their intracranial pressure and heart rate were monitored for $1 \mathrm{~h}$. Signals were continuously monitored for up to $1 \mathrm{~h}$ at an exciting frequency of $10.7 \mathrm{MHz}$. Autologous blood was administered to the experimental group, and saline to the control group (1 to $3 \mathrm{~mL}$ ) by injection of $1-\mathrm{mL}$ every $5 \mathrm{~min}$. The results showed a significant increase in MIPS as a function of the injection volume, but the heart rate was stable. In the experimental (ACH) group, there was a statistically significant positive correlation of the intracranial pressure and MIPS. The change of MIPS was greater in the $\mathrm{ACH}$ group than in the control group. This high-sensitivity system could detect a 1-mL change in blood volume. The MIPS was significantly related to the intracranial pressure. This observation suggests that the method could be valuable for detecting early warning signs in emergency medicine and critical care units.
\end{abstract}

Key words: Acute cerebral hemorrhage; Magnetic induction phase shift; Electromagnetic properties of biological tissues; Intracranial pressure

\section{Introduction}

Spontaneous acute cerebral hemorrhage $(\mathrm{ACH})$ is bleeding into the brain that occurs in the absence of trauma. Thirty-day mortality approaches $50 \%$, and only $20 \%$ of survivors achieve meaningful functional recovery at 6 months $(1,2)$. Spontaneous intracerebral hemorrhage is responsible for $10-15 \%$ of acute strokes and is associated with the highest mortality rate, with only $38 \%$ of affected patients surviving the first year (3). The study of cerebral hemorrhage has benefited from advances in medical imaging technologies such as computed tomography $(\mathrm{CT})$, magnetic resonance imaging (MRI), and positron emission tomography (PET) (4). Although such imaging devices are extremely valuable for studying cerebral hemorrhage, they are expensive and not readily available either to patients or to researchers in areas with limited economic resources $(5,6)$, emergency departments, and emergency zones. In addition, imaging techniques may be unable to detect hemorrhages when

the amount of bleeding is very small even though the potential danger is large. The goals of this study are to conduct animal experiments with our magnetic induction phase shift (MIPS) measurement device for real-time monitoring of $\mathrm{ACH}$ and to clarify the relationships of intracranial pressure (ICP), MIPS, and bleeding volume.

The relevant field of study here is that of the electromagnetic properties of biological tissues. As shown by Tarjan and McFee (7), electrodeless measurement of changing impedances in the human body can be achieved by measuring the effect of inducted eddy currents. It is well established that different biological tissues have different electromagnetic properties and can therefore be distinguished on the basis of those properties (8). The complex electrical properties of impaired tissue are substantially different from those of normal tissue, and various non-contact systems based on electromagnetic measurements have been proposed for different medical

Correspondence: M.X. Qin, College of Biomedical Engineering and Medical Imaging, Third Military Medical University, 400038 Chongqing, China. Fax: +86-023-6877-2484. E-mail: nksunj@126.com

Received January 28, 2013. Accepted October 10, 2013. First published online January 17, 2014. 
applications $(9,10)$. Magnetic induction tomography has been studied as a potential tool for the non-invasive detection of edema (9-12) in addition to other non-contact approaches for detecting electromagnetic changes in tissue (13). Measuring changes in the electromagnetic properties of tissue with two non-contacting induction coils placed near the tissue have been proposed as an alternative method to detect clinical changes in the tissue $(14,15)$. Although the information thus obtained is not in the form of an image, it can provide temporal and even gross information about local changes in tissue (16) from non-contact, volume measurements. In particular, a change in the MIPS following the occurrence of a suspected clinical episode could serve as a first-order clinical warning by detecting the presence and progression of changes in tissue (13). In previous research, we carried out experimental studies to simulate the detection of cerebral edema by using a magnetic induction method, a single detection system, and a physical model of cerebral edema $(17,18)$. We found that MIPS values were directly proportional to the volume, conductivity, and frequency. In this study, we showed that non-invasive measurements of the electromagnetic properties of the rabbit brain can be used to detect the evolution of a cerebral hemorrhage. We describe herein the fundamental principles of the technique and a prototype device. We then show results obtained from the brains of rabbits in which $\mathrm{ACH}$ was induced.

\section{Material and Methods}

\section{Theoretical basis}

The electromagnetic property that we measured in this study was the change in MIPS across time under conditions of $\mathrm{ACH}$. We considered a cylinder of tissue of radius $R$ and thickness $t$ as a case study; it was placed centrally between an excitation coil and a detection coil separated by distance $d(t<<d)$. A sinusoidal current I of angular frequency $\omega$ flowed in the excitation coil and produced a magnetic field $B$. The non-magnetic cylinder had conductivity $\sigma$ and relative permittivity $\varepsilon_{\mathrm{r}}$. The skin depth $\delta$ was assumed to be much greater than $t$, which means that the attenuation of the magnetic field $B$ produced by the cylinder was small. We used spherical polar coordinates $(r, \theta)$, as shown in Figure 1.

According to Griffiths et al. (19), at a point $P$ in the cylinder model, the magnetic field relation is given as follows.

$$
\frac{\Delta B}{B}=\left(\omega \varepsilon_{0} \varepsilon_{r}-j \sigma\right)\left(\frac{\omega \mu_{0} t d^{3}}{16}\right)\left[\frac{1}{r_{0}^{2}}-\frac{r_{0}^{2}+2 R^{2}}{\left(r_{0}^{2}+R^{2}\right)^{2}}\right]
$$

where $B$ is the primary magnetic field and $\Delta B$ is the magnetic field induced by the cylinder. Hence, $\Delta B / B$ can be defined in terms of the induced voltage in the detection coil (20) by

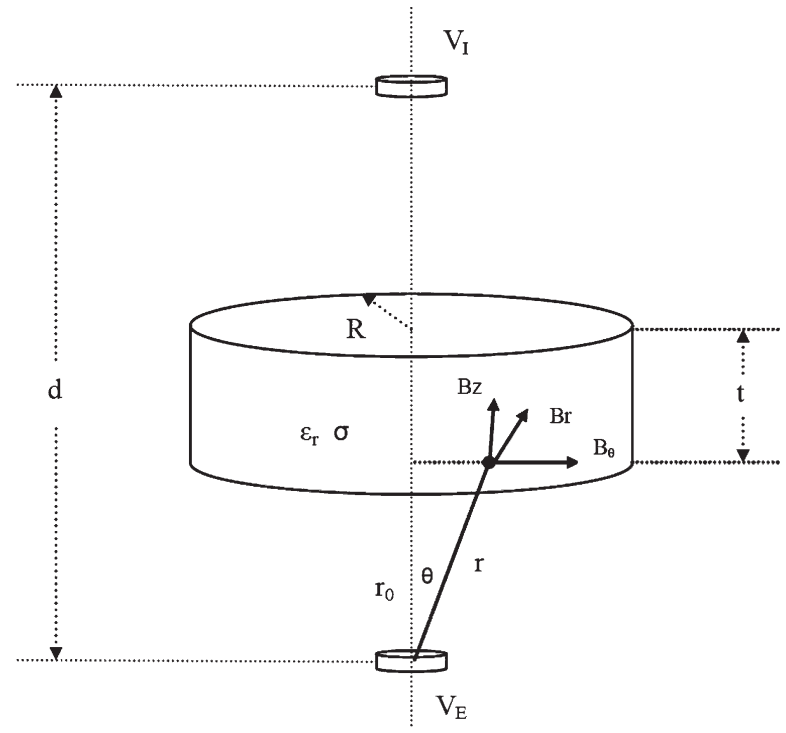

Figure 1. Magnetic field in a cylinder due to an excitation coil (shown in polar coordinates). A cylinder of radius $\mathrm{R}$ and thickness t was placed centrally and midway between a small excitation coil and a small detection coil separated by distance $d$. The cylinder had a conductivity of $\sigma$, a relative permittivity of $\varepsilon_{\mathrm{c}}$ and a permeability of $\mu_{0}$.

$\frac{\Delta B}{B}=\frac{\Delta V_{i}}{V}$

A constant $\mathrm{k}$ is defined as

$k=\left(\frac{\mu_{0} t d^{3}}{16}\right)\left[\frac{1}{r_{0}^{2}}-\frac{r_{0}^{2}+2 R^{2}}{\left(r_{0}^{2}+R^{2}\right)^{2}}\right]$

By substituting Equations 3 and 2 into Equation 1, the phase of the total induced voltage $\theta_{\mathrm{Vi}}$ in the detection coil with respect to the voltage induced by the excitation magnetic field could be expressed as a function of the frequency and electrical parameters in the cylinder between the coils (19) by

$\theta_{V i}=\operatorname{arctg}\left(\frac{\operatorname{Im}(\Delta V)}{V+\operatorname{Re}(\Delta V)}\right)=\operatorname{arctg}\left(\frac{-k \omega \sigma}{k \omega^{2} \varepsilon_{0} \varepsilon_{r}+1}\right)$

Because the phase change of current in the excitation coil was affected by the magnetic field induced by the cylinder, the phase in the excitation coil $\theta_{\mathrm{Ve}}$ could be estimated from the following expression (13)

$\theta_{\text {Ve }}=\operatorname{arctg}\left[\frac{\operatorname{Im}\left(\frac{Z_{L}+\Delta Z_{L}}{Z_{\text {out }}+Z_{L}+\Delta Z_{L}}\right)}{\operatorname{Re}\left(\frac{Z_{L}+\Delta Z_{L}}{Z_{\text {out }}+Z_{L}+\Delta Z_{L}}\right)}\right]$ 
where $Z_{L}$ represents the impedance of the excitation coil and $Z_{\text {out }}$ is the output impedance of the excitation source. The total phase shift between the voltages in the excitation and detection coils is given by the expression

$\Delta \theta=\theta_{V i}-\theta_{V e}$

The total phase shift $\Delta \theta$, which is the measured MIPS, is produced by the cylinder perturbing the excitation field. In previous simulation experiments, the MIPS (i.e., $\Delta \theta$ ) was demonstrated to be directly proportional to the volume (18); hence, we could predict the volume of $\mathrm{ACH}$ as a function of the MIPS. On the basis of the theoretical estimates, an experimental setup was built to detect $\mathrm{ACH}(17,18)$.

\section{Experimental setup to detect MIPS}

A system block diagram of the experimental setup that was built and used to detect the MIPS is shown in Figure 2. The system consists of seven modules: a signal generator, excitation coil, detection coil, phase detector, personal computer (PC), and equipment to collect and process biological signals.

The signal generator supplies a sinusoid signal $V_{\mathrm{e}}$ of approximately $5 \mathrm{~V}$ at $10.7 \mathrm{MHz}$. The resonance frequency of the generator-excitation coil system was determined experimentally to be centered on $7.65 \mathrm{MHz}$. This frequency was therefore excluded from the analysis. The range of output power from the generator was $1.5-33 \mathrm{dBm}$. The frequency stability was on the order of $10^{-4}$ and the signal-to-noise ratio in the excitation generator was $60 \mathrm{~dB}$. The excitation and detection coils were coaxially placed. Both coils comprised ten turns with a radius $r$ of $5 \mathrm{~cm}$, and the distance $d$ between the coils was $11 \mathrm{~cm}$. To avoid inductive pickup, the leads of the coils were twisted. The excitation coil induces a current in the detection coil by magnetic induction and an MIPS whose magnitude depends on the conductivity of the rabbit brain. The main components of the phase detector were two band-pass filters, two differential amplifiers, an analog-to-digital converter, a field programmable gate array chip, a digital signal processor chip, flash memory chips, and a liquid crystal display module. The phase detector could be calibrated; the temperature drift and noise could be eliminated by the calibration software in the phase detector. The performance characteristics of the phase detector were as follows: range of phase measurement, $0-180^{\circ}$; phase resolution, 0.050 ; range of gain, -10 to $35 \mathrm{~dB}$; and duration of each measurement, 3$7 \mathrm{~s}$. Measured data for a period of up to $12 \mathrm{~h}$ could be saved in the phase detector. The electrocardiograph (ECG) and ICP data collection device (6280C, Chengdu Instrument Factory, China) was controlled by a PC.

\section{Surgical procedure}

Six rabbits $(2.2-3.8 \mathrm{~kg}$, average weight $2.5 \mathrm{~kg})$ in the experimental group $(\mathrm{ACH})$ and four rabbits $(2.3-2.7 \mathrm{~kg}$, average weight $2.4 \mathrm{~kg}$ ) in a control group were anesthetized with $5 \mathrm{~mL} / \mathrm{kg}$ urethane $(25 \%)$ via injection into the ear vein. We established models of $\mathrm{ACH}$ and edema by means of stereotactic methods. The coordinates for the brain were determined using a standard atlas (21). A short midline skin incision was made and the point for a stereotactic approach through the skull determined. The head was leveled, and positioned so that the bared bregma was $1.5 \mathrm{~mm}$ higher than lambda. With the position of the bregma as the reference point, the coronal zero plane (AP0) passed through the coronal suture. A1 and $\mathrm{P} 1$ were coronal planes $1 \mathrm{~mm}$ anterior and $1 \mathrm{~mm}$ posterior, respectively, to AP0. The scope of the internal capsule was between $A 5$ and $P 2$. We chose the P1 coronal plane to make a puncture point on the right

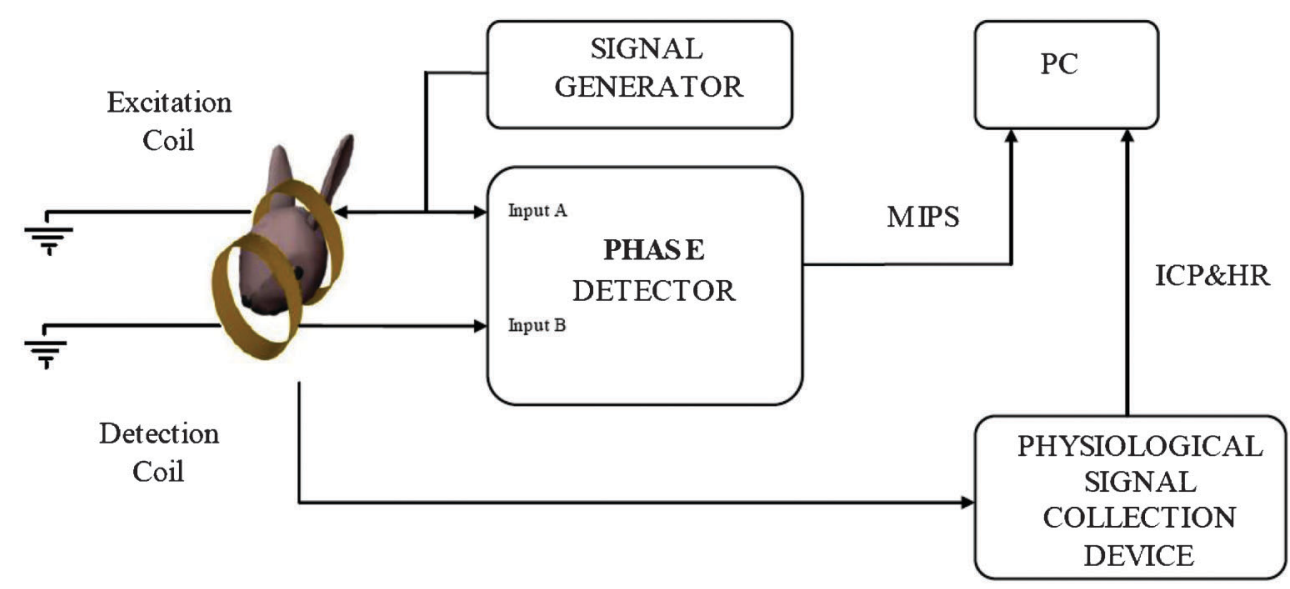

Figure 2. System block diagram of the magnetic induction phase shift (MIPS) detection method. Data from rabbits were obtained with a physiological signal collection device. Intracranial pressure (ICP), heart rate (HR), and MIPS were synchronously collected to compare the measured results. 
coronal suture $(\mathrm{R}=6 \mathrm{~mm})$ and posterior to the sagittal suture $(P 1, A P=1 \mathrm{~mm})$. A 1-mm drill bit was twisted by hand to perforate the skull. A needle $(\mathrm{d}=0.7 \mathrm{~mm})$ was then introduced to the proper depth $(\mathrm{H}=13 \mathrm{~mm})$. Autologous blood (1 to $3 \mathrm{~mL}$ ) was slowly injected in the $\mathrm{ACH}$ group and the same volume of normal saline was injected in the control group. A physiological signal collection device (RM6280C, Chengdu Instrument Factory) was used to measure the changes in the ICP of rabbit brain (22) and heart rate (HR). As shown in Figure 3, rabbits were operated to monitor ICP and HR.

The Animal Experiments and Ethical Committee of our university approved all experimental protocols and the care of animals was carried out in accordance with the Declaration of Helsinki and International Association for the Study of Pain (IASP) guidelines $(23,24)$.

\section{Data processing and analysis}

Data from the rabbits were obtained with a physiological signal collection device, and showed that HR, ICP and HR were stable throughout the experimental procedure. The MIPS signals recorded by the phase detector were collected synchronously with the ICP and ECG data acquired by the physiological signal collection device.

Figure 4 shows the experimental setup for detecting cerebral hemorrhage in the rabbits in the experiment group. ICP, HR, and MIPS were collected synchronously for comparison of the measured results; changes in the MIPS were recorded and compared with baseline measurements obtained after the surgical procedure. Experimental excitation and detection coils were coaxially placed around the heads of the rabbits. In all experiments, the coils were placed so that the head was centered between the excitation and detection coils. Changes in MIPS were recorded as a function of the injection volume. The key study hypothesis was that the changes in MIPS measured with the detection coil would reflect the changes in electrical conductivity of the brain.

Baseline data obtained from a single $\mathrm{ACH}$ rabbit after the surgical procedure $(t=0)$ in a typical experiment are reported in Figure 5. Signals were continuously monitored for $1 \mathrm{~h}$, during which a total of $3 \mathrm{~mL}$ autologous blood was injected by administration of three 1-mL injections given at 5-min intervals. The injection points are shown by red vertical bars. After each injection, the phase detector measured the MIPS for 5 min, collecting 70 data points. We selected 10 stable, continuous MIPS measurements before each subsequent injection, as shown by red circles. Overall, the MIPS and ICP showed an increasing trend as the injection volume increased. Some fluctuations in the MIPS due to the movement of rabbits were observed. These unstable data points were excluded from the data evaluation. The ICP and HR data were selected at the same times as the 10 MIPS measurements. Each data set was then averaged to eliminate interference in the data. The same data collection and processing methods were used with the control group.

\section{Results and Discussion}

The average ICP and MIPS values obtained from 6 $\mathrm{ACH}$ rabbits at an excitation frequency of $10.7 \mathrm{MHz}$ are reported in Figure 6 . As the injection volume increased from 1 to $3 \mathrm{~mL}$, the changes in both MIPS and ICP also increased. The slope of each curve was stable, and changes in both the MIPS and ICP were linearly related to the injection volume. The slope of each function reflected the sensitivity level. These results demonstrated that the MIPS method was a satisfactory, reliable, and feasible means to detect and monitor ACH. The MIPS system could detect a small amount of bleeding in the brain, with sensitivity higher than that of detecting changes in the ICP. Both the ICP and the MIPS increased with the

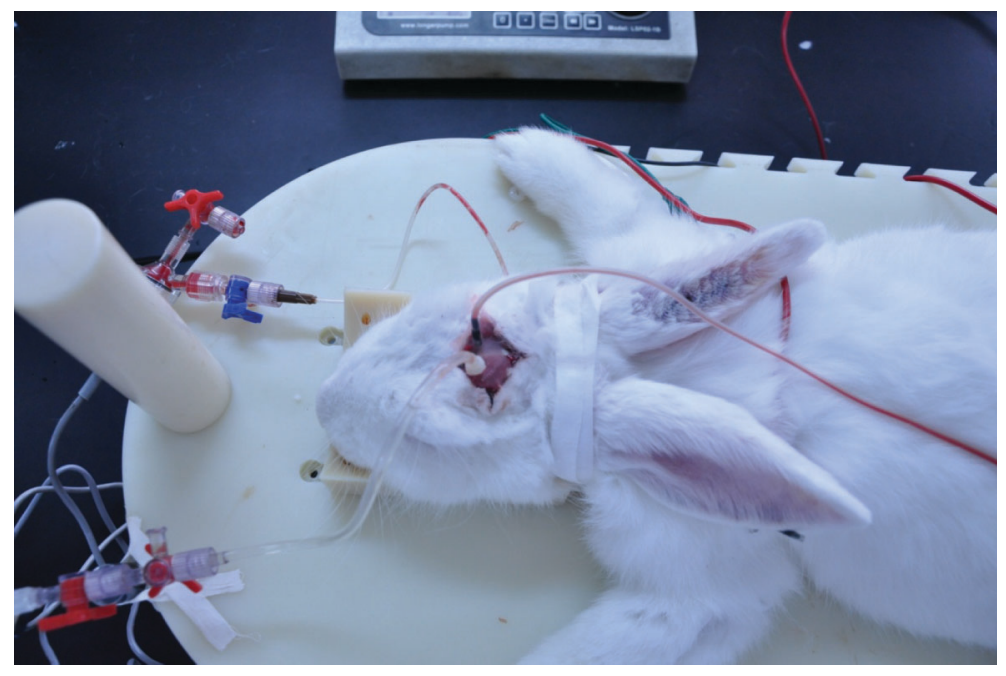

Figure 3. Rabbit model of acute cerebral hemorrhage. The model was established by means of stereotactic methods. 


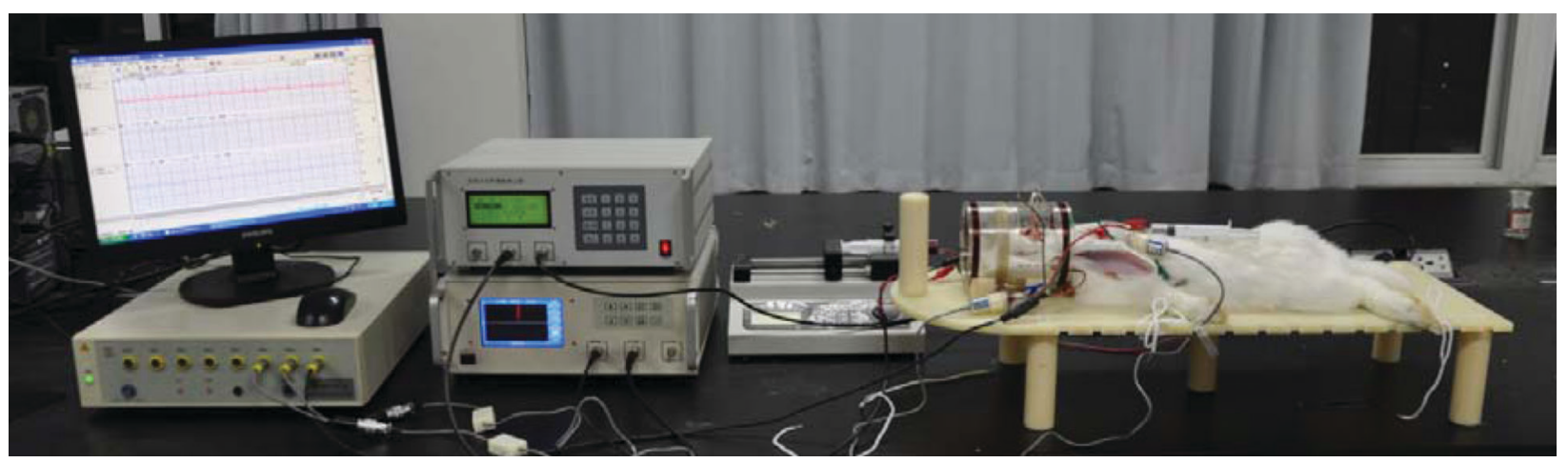

Figure 4. Experimental setup to detect acute cerebral hemorrhage in rabbits with the magnetic induction phase shift method. The excitation and detection coils were coaxially placed.

bleeding volume, and a change in the MIPS suggested a change in the ICP because the latter was directly proportional to the MIPS. A change in the MIPS might reflect changes in the cerebrospinal fluid and cerebral blood volumes, as reported previously in the literature (13). Our MIPS system is a non-invasive, non-contact method for detection of the volume of cerebral hemorrhage.

Increase of either edema or $\mathrm{ACH}$ can cause an increase of ICP. As shown in Figure 7, the MIPS in the $\mathrm{ACH}$ group was significantly different from that of the control group. The MIPS data were analyzed in rabbits with stable HRs and vital signs. The average MIPS values in the $6 \mathrm{ACH}$ rabbits in the experimental group increased to $0.6173 \pm 0.1976$ degrees as the injection volume increased from 0 to $3 \mathrm{~mL}$. Figure 7 also shows that MIPS changed from 0 to $0.041 \pm 0.0073$ degrees for 4 rabbits from the control group due to the increase of edema volume. The change of MIPS in the experimental group was greater than in the control group. These results were expected with the MIPS method because the conductivity of saline $(0.281 \mathrm{~s} / \mathrm{m})$ is less than the conductivity of blood $(1.101 \mathrm{~s} / \mathrm{m})$ at excitation frequency 10.7 $\mathrm{MHz}$ (17). The average conductivity of the brain during $\mathrm{ACH}$ is also less than that seen in brains with edema. The observed changes in conductivity reflect changes in MIPS, consequently the change of MIPS observed in the $\mathrm{ACH}$ group should be greater than that in the edema group.

Figure 6 also shows that the functional relationships of ICP and MIPS to the injection volumes were not identical. The slope of the MIPS was greater than that of the ICP during the early stages of hemorrhage, but this was reversed during later stages. This finding suggested that MIPS measurements would be useful as an early warning of an increase in ICP. Possible reasons for the differences in the observed behavior of ICP and MIPS include volume errors due to manual blood injection and physiological regulation of the cerebral blood flow (CBF) and cerebrospinal fluid (CSF).

Owing to damage of the skull and brain, cerebral hemorrhage and cerebral edema, the blood volume flow

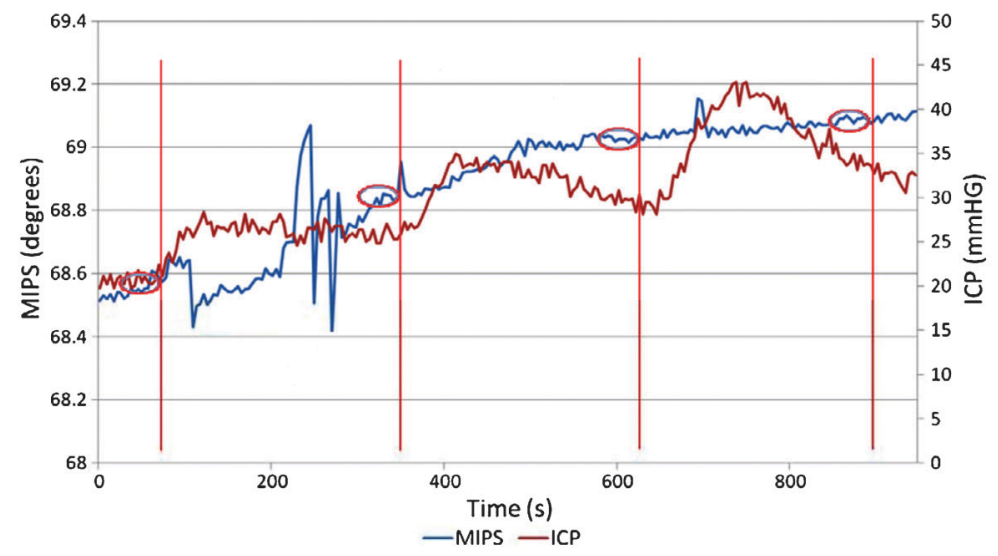

Figure 5. Data from one rabbit with acute cerebral hemorrhage obtained with the magnetic induction phase shift (MIPS) experimental setup and the physiological signal collection device. The MIPS increased from $68.5^{\circ}$ to $69.1^{\circ}$ (blue tracing) whereas the intracranial pressure (ICP) increased from 18 to $33 \mathrm{mmHg}$ (red tracing). The red vertical bars indicate injection points, and red circles indicate data selection. 


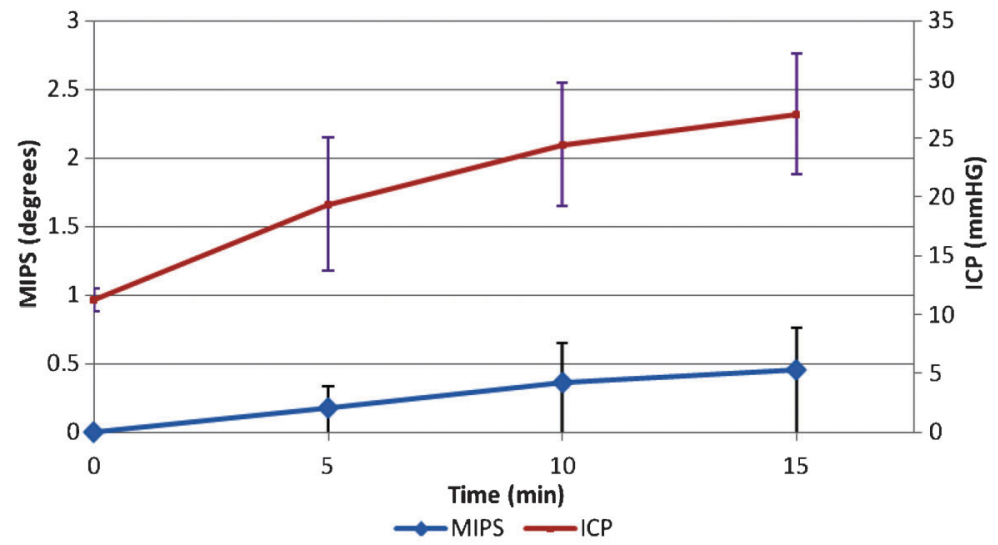

Figure 6. Average intracranial pressure (ICP) and magnetic induction phase shift (MIPS) of 6 rabbits with acute cerebral hemorrhage as a function of time after the surgical procedure. The total injection volume increased from 0 to $3 \mathrm{~mL}$ during the 15-min period. rate in the animals used in the study was approximately 100-300 $\mu \mathrm{L}$ (due to individual differences) within less than $1 \mathrm{~h}$ after the craniotomy. An animal hemorrhage model should take spontaneous intracerebral hemorrhage into consideration. The cerebral perfusion pressure remained largely unchanged.

We plan to use the NI-PXI design virtual instrument to improve the speed of the phase detector and ultimately achieve real-time monitoring $(<5 \mathrm{~ms})$ of the MIPS. We also plan to test different excitation frequencies as well as settings for the compensation coils. To improve this experimental rabbit model, we will need to examine different bleeding positions, means of inducing cerebral edema and hemorrhage, and to use large study populations. The use of imaging methods to demonstrate the physiological regulating effects of CSF and CBF is necessary. For clinical applications, we need to ensure that the method meets safety regulations; furthermore, we will determine the optimal frequency and power. We plan to recruit more than 30 individuals to participate in a planned clinical study.

This study is the first to use animal experiments to demonstrate the relationship between ICP and changes of
MIPS. A comparison of the analytical and experimental results to those of previous studies $(14,15)$ suggests that various physiological conditions in the brain, such as edema and ischemia, can be distinguished by analyzing the change in MIPS at specific frequencies. In this study, the MIPS curves were significantly related to the ICP curves. This observation suggests that in addition to continuous monitoring, the proposed method could be valuable for detection of early warning signs in emergency medicine and critical care units.

In future studies, we plan to optimize our system and the experimental scheme according to these results. We plan to perform up to $12 \mathrm{~h}$ or more of long-term monitoring. The inductance of the coils, and electrical conductivity of the rabbit brain were not calculated. Owing to time constraints, the sample size was small and there was no control group using traditional methods such as CT, MRI, or PET imaging. Further developments will be directed towards contrast research with established clinical methods.

Measuring changes in the MIPS as a function of volume after brain injury has the potential to become a simple method for early non-contact detection of the

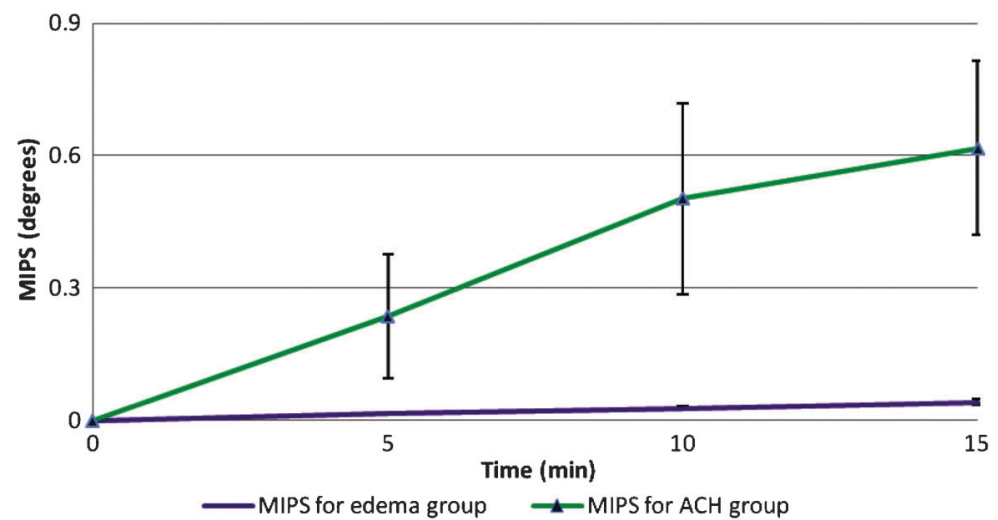

Figure 7. Magnetic induction phase shift (MIPS) of the acute cerebral hemorrhage $(\mathrm{ACH})$ group compared with the control group. The average value of MIPS of 6 rabbits from the ACH group increased to $0.6173 \pm 0.1976$ degrees with the injection volume from 0 to $3 \mathrm{~mL}$. MIPS changed from 0 to $0.041 \pm 0.0073$ degrees in the control group. 
occurrence and progression of cerebral hemorrhage. Such a technique would certainly be useful in emergency medicine, critical care, and other clinical applications. In addition, the measuring device can be small and portable. This study is the first to clarify the relationships of ICP and MIPS to bleeding volume by using magnetic induction. Because of the high sensitivity of MIPS measurements in the early stages of $\mathrm{ACH}$, the method could be valuable for detecting early warning signs. Further studies

\section{References}

1. Wagner KR, Broderick JP. Hemorrhagic stroke: pathophysiological mechanisms and neuroprotective treatments. In: Lo EH, Marwah J (Editors), Neuroprotection. Scottsdale: Prominent Press; 2001. p 471-508.

2. Naval NS, Nyquist PA, Carhuapoma JR. Management of spontaneous intracerebral hemorrhage. Neurosurg Clin N Am 2008; 19: 415-423, v, doi: 10.1016/j.nec.2008.07.014.

3. Qureshi Al, Tuhrim S, Broderick JP, Batjer HH, Hondo H, Hanley DF. Spontaneous intracerebral hemorrhage. N Engl J Med 2001; 344: 1450-1460, doi: 10.1056/NEJM200105 103441907.

4. Kidwell CS, Chalela JA, Saver JL, Starkman S, Hill MD, Demchuk AM, et al. Comparison of MRI and CT for detection of acute intracerebral hemorrhage. JAMA 2004; 292: 1823-1830, doi: 10.1001/jama.292.15.1823.

5. Otten DM, Onik G, Rubinsky B. Distributed network imaging and electrical impedance tomography of minimally invasive surgery. Technol Cancer Res Treat 2004; 3: 125-134.

6. Granot $Y$, Ivorra A, Rubinsky B. A new concept for medical imaging centered on cellular phone technology. PLOS One 2008; 3: e2075, doi: 10.1371/journal.pone.0002075.

7. Tarjan PP, McFee R. Electrodeless measurements of the effective resistivity of the human torso and head by magnetic induction. IEEE Trans Biomed Eng 1968; 15: 266278, doi: 10.1109/TBME.1968.4502577.

8. Foster KR, Schwan HP. Dielectric properties of tissues and biological materials: a critical review. Crit Rev Biomed Eng 1989; 17: 25-104.

9. al-Zeibak S, Saunders NH. A feasibility study of in vivo electromagnetic imaging. Phys Med Biol 1993; 38: 151-160, doi: 10.1088/0031-9155/38/1/011.

10. Korjenevsky AV, Cherepenin VA. Magnetic induction tomography. J Commun Technol El+ 1997; 42: 469-474.

11. Korjenevsky AV, Cherepenin VA. Progress in realization of magnetic induction tomography. Ann N Y Acad Sci 1999; 873: 346-352, doi: 10.1111/j.1749-6632.1999.tb09482.x.

12. Griffiths $\mathrm{H}$. Magnetic induction tomography. Meas Sci Technol 2001; 12: 1126-1131, doi: 10.1088/0957-0233/12/8/319.

13. Gonzalez CA, Villanueva C, Vera C, Flores O, Reyes RD, Rubinsky $B$. The detection of brain ischaemia in rats by inductive phase shift spectroscopy. Physiol Meas 2009; 30: will be required to confirm the observations of this preliminary study.

\section{Acknowledgments}

Research supported by the National Natural Science Foundation of China (\#61072254) and the Science and Technology Research Plan Project Foundation of Chongqing (\#CSTC2012GG-YJS10013).

809-819, doi: 10.1088/0967-3334/30/8/006.

14. Gonzalez CA, Rubinsky B. A theoretical study on magnetic induction frequency dependence of phase shift in oedema and haematoma. Physiol Meas 2006; 27: 829-838, doi: 10.1088/0967-3334/27/9/006.

15. Gonzalez CA, Rubinsky B. The detection of brain oedema with frequency-dependent phase shift electromagnetic induction. Physiol Meas 2006; 27: 539-552, doi: 10.1088/ 0967-3334/27/6/007.

16. Rojas R, Rubinsky B, Gonzalez CA. The effect of brain hematoma location on volumetric inductive phase shift spectroscopy of the brain with circular and magnetron sensor coils: a numerical simulation study. Physiol Meas 2008; 29: S255-S266, doi: 10.1088/0967-3334/29/6/S22.

17. $\mathrm{Xu} \mathrm{L}$, Qin MX, Jin G. Study of PSSMI for cerebral hemorrhage detection: an experimental simulation. 4th International Congress on Image and Signal Processing. October 15-17, 2011, Shangai. p 266-268.

18. Jin $G$, Qin MX, Wang C. Experimental study on simulated cerebral edema detection with PSSMI. Commun Systems Inform Technol LNEE 2011; 100: 361-367, doi: 10.1007/ 978-3-642-21762-3 47.

19. Griffiths H, Stewart WR, Gough W. Magnetic induction tomography. A measuring system for biological tissues. Ann N Y Acad Sci 1999; 873: 335-345, doi: 10.1111/j.17496632.1999.tb09481.x.

20. Scharfetter H, Casanas R, Rosell J. Biological tissue characterization by magnetic induction spectroscopy (MIS): requirements and limitations. IEEE Trans Biomed Eng 2003; 50: 870-880, doi: 10.1109/TBME.2003.813533.

21. Sawyer $\mathrm{CH}$, Everett JW, Green JD. The rabbit diencephalon in stereotaxic coordinates. J Comp Neurol 1954; 101: 801824, doi: 10.1002/cne.901010307.

22. Jing $\mathrm{WL}$, Zhang $\mathrm{XQ}$, Jin $\mathrm{XL}$. Study on the model building of internal capsule hemorrhage and the changes of intracranial pressure and discharge of vagus nerve in rabbits. Shanxi Med J 2007; 36: 18-21.

23. Declaration of Helsinki. Brit Med J 1996; 313: 1448-1449.

24. Zimmermann M. Ethical guidelines for investigations of experimental pain in conscious animals. Pain 1983; 16: 109110, doi: 10.1016/0304-3959(83)90201-4. 